\title{
The fitness threshold model: Random environmental change alters adaptive landscapes
}

\author{
THOMAS J. DeWITT ${ }^{1 *}$ and JIN YOSHIMURA ${ }^{2}$ \\ ${ }^{1}$ Center for Ecology, Evolution and Behavior, T.H. Morgan School of Biological Sciences, University of Kentucky, Lexington, \\ KY 40506-0225, USA and ${ }^{2}$ Department of Systems Engineering, Shizuoka University, Hamamatsu 432, Japan
}

\begin{abstract}
Summary
We used a probabilistic optimization model to explore the joint evolutionary effects of random phenotypic and environmental variation. Two forms of environmental noise were defined in which the optimal phenotype remained constant but all organisms experienced either the same proportionate or the same absolute fitness gains and losses. There was no evolutionary effect of proportionate fitness fluctuations. In contrast, the optimal genotype varied with absolute fitness fluctuations, despite the environmental effect being phenotypeindependent. We refer to such phenotype-independent fluctuation in absolute fitness as the 'fitness threshold model', because shared fitness effects determine the zero-fitness points (i.e. the baseline) on an intrinsic fitness function. Thus, environmental effects that are unrelated to a focal trait can cause peak shifts in the genetic optimum for the trait. Changes in the fitness threshold not only changed peak locations, but also altered the slopes defining the peaks, and so should alter the rate of evolution towards optima. This model pertains to evolution in any system, unless there is no phenotypic or environmental variance, or the selection function and distribution of phenotypic error assume similar shapes. Our results have many basic and applied implications for topics such as the maintenance of genetic variation, the canalization of development and the management of natural populations.
\end{abstract}

Keywords: development; evolution; optimality; phenotypic variation; stochastic environments

\section{Introduction}

A basic observation about natural populations is that they fluctuate with changing environmental pressures (Ford, 1964; Hassell, 1978). Environmental variation in nature has led to the evolution of traits such as phenotypic plasticity, seed-banking, learning, habitat choice, risk-sensitive behaviour, dispersal and others. It is hard to think of organisms without at least some of these traits. Therefore, a core problem in predictive evolutionary models is to understand the effects of environmental variation on evolution.

Environments vary in many ways. Directional environmental change in which the optimal phenotype shifts monotonically has obvious evolutionary consequences (sustained directional selection; Ford, 1964; Dobzhansky, 1970; Mayr, 1970). When environments fluctuate such that phenotypic optima shift arbitrarily from generation to generation, evolution may favour any of a number of outcomes, such as genetic polymorphism (Levins, 1968), intermediate (generalist) genotypes (Levins, 1968), environmental tolerance (Slatkin and Lande, 1976), bet-hedging (Seger and Brockmann, 1987) or phenotypic plasticity (Via and Lande, 1985). We did not include these forms of environmental fluctuation in our models because they have received considerable attention already.

Environmental fluctuation in which the optimal phenotype remains constant has not been widely considered. In this paper, we address two forms of fluctuation in which the phenotypic optimum

\footnotetext{
* Author to whom all correspondence should be addressed.
} 
remains the same because fitness effects of environmental shifts are experienced similarly by all individuals (i.e. at random with respect to the phenotype). Fluctuation such as this is commonly viewed as 'environmental noise' and is not traditionally thought to be important in evolution (Mayr, 1970; Weis and Gorman, 1990). If genes experience selection only through their phenotypic effects, and environmental shifts produce only phenotype-independent fitness effects, then how can selection among genotypes occur?

The models developed below address the question of whether environmental noise can create alternative genotypic optima despite the constancy of a phenotypic optimum. We compare the results of models with and without environmental variation and then discuss the evolutionary, empirical and applied implications of our results.

\section{Sources of variation in fitness}

\section{Phenotypic variation within generations}

The fitness of a genotype depends upon the phenotypes that are produced by development (Waddington, 1957; Dobzhansky, 1970). Phenotypes are not produced precisely, however, because deviations are induced by fluctuations in the environment that affect development (Fisher, 1930; Schmalhausen, 1949; Waddington, 1957). In some cases, selection favours developmental canalization and hence decreases phenotypic variance (Schmalhausen, 1949; Waddington, 1957; Weis and Gorman, 1990). In other cases, selection favours phenotypic variance (Slatkin and Lande, 1976; Bulmer, 1984; Bull, 1987; Seger and Brockmann, 1987; Gabriel and Lynch, 1992). Either way, development is never perfectly canalized, so that each genotype effectively specifies a mean phenotype with a characteristic distribution of error (Dobzhansky, 1970; Yoshimura and Shields, 1987). We use the term 'error' in the statistical sense of deviations from an average value, not in the sense of mistakes.

The probabilistic nature of gene expression can prevent selection from favouring genotypes that produce mean phenotypes matching the phenotypic optimum (Mountford, 1968; Yoshimura and Shields, 1987). This effect of phenotypic variation occurs to the degree that the fitness function and the distribution of phenotypic error are dissimilar. This point is key to understanding the present models. Consider an asymmetric fitness function for a phenotypic character, $z$, and a series of genotypes producing normally distributed phenotype distributions with mean $\mu_{i}$ and standard deviation $\sigma$ (Fig. 1). Let us ask which genotype has the highest fitness. The expected fitness of a genotype is the product of the phenotype distribution that it produces and the fitness function (Yoshimura and Shields, 1987, 1992, 1995). Although genotype 2 in the figure most commonly produces the phenotype with maximum fitness, the fitness expectation of genotype 1 is highest overall. Given the shape of the fitness function, which is truncated on the right, phenotypic error made to the right (higher $z$ ) receives lower fitness than the equivalent error made to the left. Thus, phenotype distributions that allow for the asymmetric consequences of phenotypic error are favoured.

Ideally, studies will emerge that include estimates of the shape of fitness functions (reviewed by Brodie et al., 1995) and the shape of phenotype distributions from single genotypes. There is currently little information available to tell how often fitness functions and phenotype distributions from single genotypes are similar or dissimilar. It seems reasonable that they will often differ. The notion of a stochastic environment implies that alternative fitness functions apply each generation, preventing matching of fitness function and phenotype distribution shapes. If the distribution of phenotypic error is determined by genetic and developmental constraints, then it is likely to change only gradually across many generations. It is not necessary in the models below that one function 


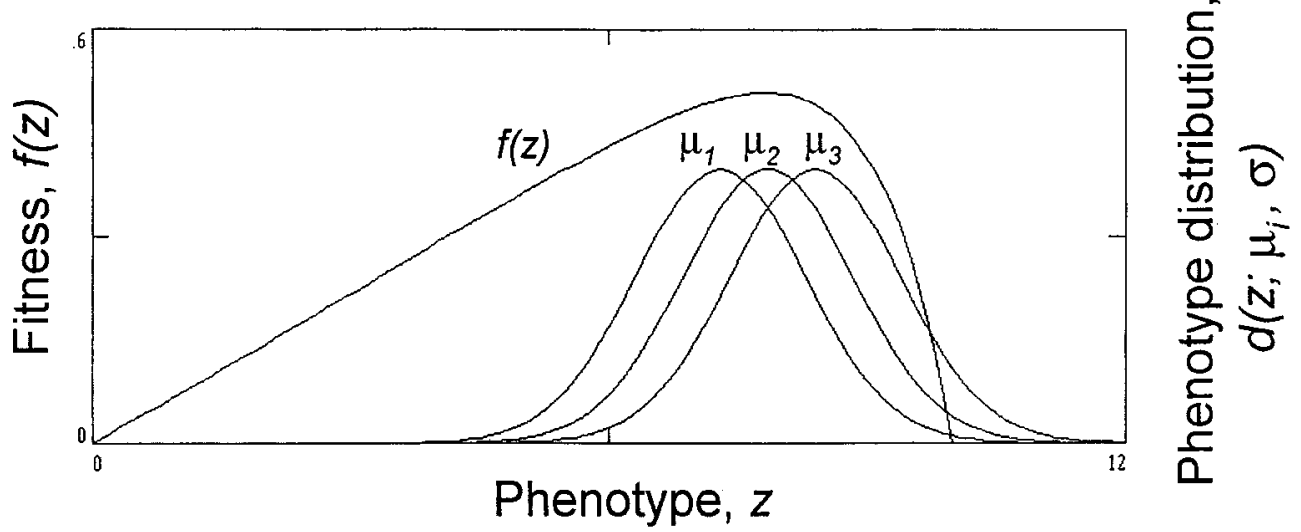

Figure 1. A fitness function and three genetically coded phenotype distributions. Fitness is a function of the phenotypic value, $f(z)$. The phenotype distributions produced by the genotypes are represented by their mean values, $\mu_{i}$. The fitness expectations for each genotype cannot be based on its mean alone but must be calculated by integrating fitness over the entire distribution of possible phenotypes. Relative fitness expectations are $1.000,0.975$ and 0.886 for the phenotype distributions from genotypes 1,2 and 3 respectively. Although the mean phenotype produced by genotype 2 has the maximum possible fitness, the distribution of phenotypes produced by genotype 1 has the highest overall fitness expectation. This is true because the tail of distribution 2 extends into phenotype space with zero fitness, implying that phenotypes produced by genotype 2 would often not reproduce. Hence, the optimal phenotype distribution $\left(\mu_{1}\right)$ is risk-averse compared to the distribution whose mean achieves the optimum.

be symmetric and the other be asymmetric. We build this structure into our models as an intuitive convenience. Evolutionary biologists frequently assume that phenotypic error is normally distributed around breeding values (Fisher, 1930; Falconer and Mackay, 1996) and well-resolved fitness functions tend to be asymmetric (see Roff, 1981; Boyce and Perrins, 1987; Weis et al., 1992).

\section{Environmental change among generations}

Environments vary within and among generations (Levins, 1968). Within-generation environmental variation is implicit in our models, as it is assumed to contribute to developmental error and hence to phenotypic variance (Fisher, 1930; Schmalhausen, 1949; Waddington, 1957; Dobzhansky, 1970). Now we consider the effects of among-generation environmental variation, which is likely for most species (Ford, 1964; Mayr, 1970). For example, high- and low-pest years, or cycles, create generational selection on plant and animal populations (Hassell, 1978).

The fitness effects of environmental change can be visualized by initially defining a default fitness function for a focal trait, $f(z)$. The shape and form of $f(z)$ is then modified to reflect environmental changes. For our purposes, two random (i.e. phenotype-independent) forms of environmental change can be specified in which the same proportionate, or the same absolute, change in fitness applies for all individuals in the population, regardless of their phenotype and, hence, regardless of their genotype. If the same proportionate decline in fitness is applied for all phenotypes, the original fitness function appears to deflate (Fig. 2B). If the same absolute fitness value is deducted for all phenotypes, the fitness function appears to be lower along the y-axis (Fig. 2C; see also Leon, 1993). Note that neither form of change alters the phenotypic optimum within a generation. Thus, the evolutionary effects of these environmental changes, if any exist, are not immediately obvious. 

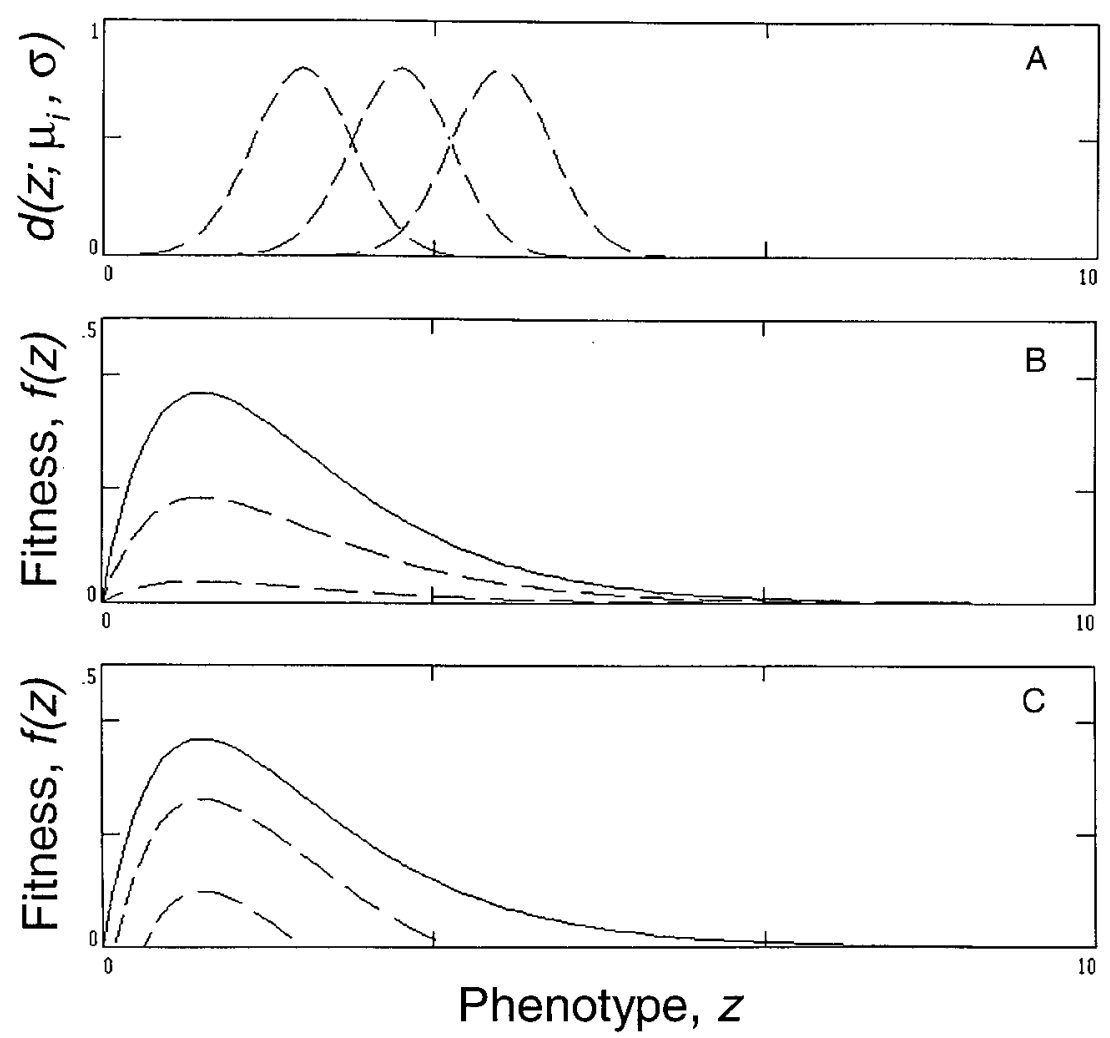

Figure 2. Sources of variation in fitness. (A) Genotypes specify phenotype distributions due to phenotypic error. Each distribution represents a genetically coded mean phenotype, $\mu$, with $\sigma=1$. (B) Fitness functions showing proportionate fitness changes (PFC). The areas under the two lower curves are 0.5 and 0.1 of the upper curve. In the PFC model, the lower curves represent harsher environments, such that all individuals lose the same proportion of their fitness, regardless of their phenotype. (C) Fitness functions showing absolute fitness change (AFC). The relative areas below the functions are 1.0, 0.5 and 0.1 . The AFC model involves environmental change that is uncorrelated with the phenotypic value, $z$, such that all individuals lose the same absolute amount of fitness. The mechanism of the AFC is the fitness threshold described in the text and in Fig. 3 .

Although both forms of environmental change may occur simultaneously, we model each separately to isolate and contrast their independent effects. To emphasize, we do not model other forms of variation, such as fitness functions shifting to the left or right, because we are interested only in the effects of environmental noise (i.e. the optimal phenotype does not change).

In Fig. 2B, fitness is proportionately altered for all phenotypes. We refer to this environmental change as 'proportionate fitness change' (PFC). As an example, consider an annual plant species. Each plant has an intrinsic fitness defined by a phenotypic value such as size, $w=f$ (size). However, if fire destroys plants regardless of their size, then the expected fitness of the plants is the intrinsic fitness based on phenotype, discounted by the chance of fire, $w=f($ size $) \cdot(1-$ prob[fire]). The higher curve in Fig. 2B represents a fire-safe year; the lower curve represents a fire-prone year.

In Fig. $2 \mathrm{C}$, fitness is reduced by the same absolute value for all individuals in the population, regardless of their value of $z$. We refer to this type of environmental change as 'absolute fitness 
change' (AFC). The general mechanism of this model is a change in the fitness threshold, as illustrated in Figs $3 \mathrm{~A}-\mathrm{C}$. For example, a phenotype that contributes 0.9 fitness units would not be favoured over one that contributes 0.6 units if the fitness threshold is over 0.9 , simply because neither phenotype would reproduce anyway. Only fitness contributions from the character $z$ that are higher than 0.9 (the fitness threshold) would be relevant to evolution. As a specific example, consider two temperate freshwater fish of a given size. Fish A has $100 \mathrm{~g}$ body reserves of fat, whereas fish B has $90 \mathrm{~g}$ due to differences in jaw morphology. Each fish must burn $50 \mathrm{~g}$ of fat in a mild winter to survive to reproduce, leaving 50 and $40 \mathrm{~g}$ body fat, respectively, to reproduce. The maintenance cost (50 g in a mild winter) is independent of jaw morphology. In a harsh winter, each fish must burn $80 \mathrm{~g}$ of fat, leaving 20 and $10 \mathrm{~g}$ body fat to produce offspring. Fish B is disfavoured in either type of winter, but its realized fitness relative to fish $\mathrm{A}$ is 0.8 in a mild winter and 0.5 in a harsh winter. Although both fish pay the same maintenance cost, the relative fitness differences between fish changes. Thus, the intrinsic shape of the fitness function is determined by jaw morphology, whereas the maintenance cost sets the baseline (fitness threshold) for the function. The lower curve in Fig. 2C represents a harsh-winter year; the upper curve represents a mild-winter year. Symbolically, $w=f($ jaw shape $)-v$, where $v$ is the absolute fitness decrement of the maintenance cost.

We can now address the evolutionary outcome of the two forms of environmental change (PFC and $\mathrm{AFC}$ ) using probabilistic optimization methods. We are interested in a general biological scenario in which: (1) any of a number of mechanisms produce phenotypic variation within generations; (2) there is a mismatch in shape between phenotype distributions and fitness functions; and (3) environmental fluctuations occur among generations. Our goal is to demonstrate as simply as possible whether environmental noise can affect evolution.

\section{Models}

Here we contrast the forms of environmental change shown in Figs 2B and 2C (PFC and AFC, respectively). We assume that each genotype (i) codes for a normal phenotype distribution with
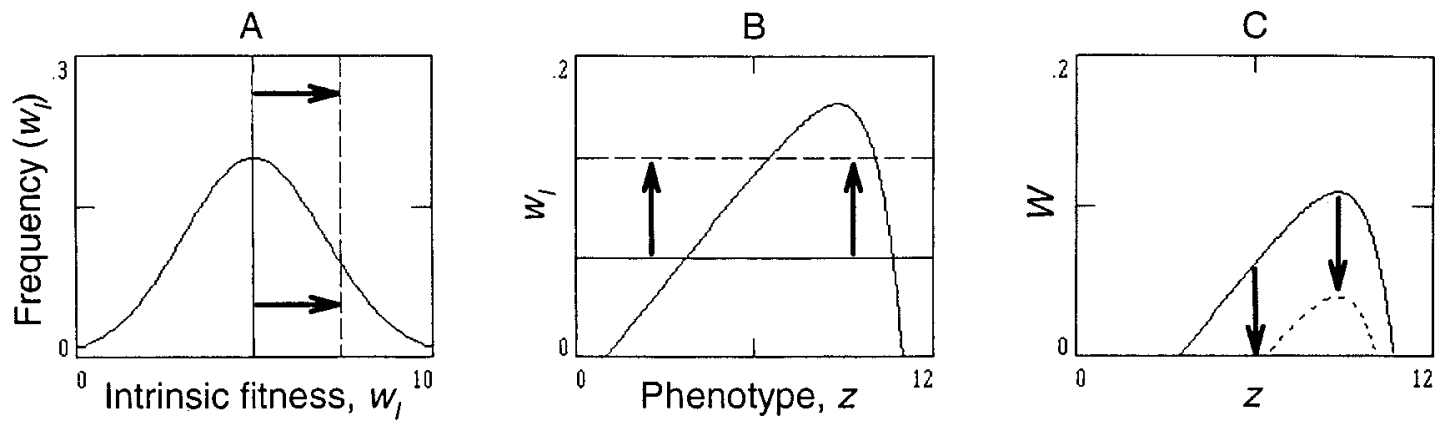

Figure 3. Fitness threshold model. (A) Only individuals above a certain intrinsic fitness survive and reproduce. Arrows indicate an environmental shift that increases the threshold so that only a fitter subset of individuals survive. Intrinsic fitness is defined phenotypically, whereas the position of the threshold is defined by an arbitrary environmental factor. (B) Effect of the raised fitness threshold on a fitness function. Only the higher contributions of the trait $z$ to fitness are now relevant to evolution; fitness is 0 for any value of $z$ that does not contribute the threshold level of fitness. The arrows indicates the threshold shift. With the lower threshold, a phenotype of $z$ $=5$ is favoured over $z=6$, whereas with the higher threshold, both phenotypes fail to reproduce. (C) Fitness functions from panel (B) with the fitness thresholds standardized (i.e. the x-axis is the threshold). 
mean $\mu_{i}$ and standard deviation $\sigma$ (Fig. 2A). In each model, we solve for the optimal phenotype distribution, which is referred to as the genetic optimum (Yoshimura and Shields, 1995). The genetic optimum is represented by the mean phenotype it produces, $\mu^{*}$. The genetic optimum often differs from the phenotypic optimum, $z^{*}$, which is not an optimal distribution but is simply the optimal trait value. (Optimal values are indicated throughout this paper by asterisks.)

To obtain the fitness expectation for a genotype within a generation, we integrated the product of the phenotype distribution and the fitness function (Yoshimura and Shields, 1987, 1992, 1995):

$$
w\left(\mu_{i j}\right)=\int f(z)_{j} \cdot d\left(z ; \mu_{i}, \sigma\right) d z
$$

where $w\left(\mu_{i j}\right)$ is the fitness of genotype $i$ in environment $j$. The function $f(z)_{j}$ describes fitness of trait $z$ in environment $j$, whereas $d\left(z ; \mu_{i}, \sigma\right)$ is a normal probability distribution of phenotypes from genotype $i$.

Empirically derived fitness functions are generally asymmetric (e.g. Roff, 1981; Boyce and Perrins, 1987; Weis et al., 1992). Rather than choose an empirical function, we selected the gamma distribution, $\gamma(z ; v, \alpha)$, as our default fitness function because distributions are computationally simple to work with (e.g. there is no need to calculate $\mathrm{x}$-intercepts for integration):

$$
f(z)_{1}=\gamma(z ; v, \alpha)=\frac{\alpha^{v}}{\Gamma(v)} \cdot z^{v-1} \cdot \mathrm{e}^{-\alpha \cdot z}
$$

The choice of function shape is arbitrary; however, the $\gamma$ distribution (setting $v=2, \alpha=1$ ) is very similar to a recent function derived by Oksanen and Lundberg (1995) for foraging time in mammals. It is only necessary for our purposes to choose an asymmetric function as a clear contrast to the normal curve that is frequently assumed for phenotypic error.

Having selected a default fitness function to represent an arbitrary 'good' environment $(j=1$, default), we derived fitness functions in harsher environments $(j=2$, modified) for both the PFC and AFC models by reducing the area under the $\gamma$ distribution to a proportion, $A$, of the original area. Figures $2 \mathrm{~B}$ and $2 \mathrm{C}$ illustrate the $\gamma$ function $(A=1 ; j=1)$ and two harsher environments $(j=2)$ where $A$ is 0.5 or 0.1 . For the PFC model, we created proportionate fitness declines by multiplying $\gamma \cdot A$. For the AFC model, we subtracted the same value at every point along the original curve, such that the area under the curve was $A$ of its original value. That is, we raised the fitness threshold to create absolute fitness declines.

For each model we contrasted the genetic optimum under conditions that fluctuated among generations from the good environment to a harsher environment. We let $p$ represent the frequency of generations that experienced good environments $(j=1=\gamma) ; 1-p$ was the frequency of generations experiencing the modified environments $(j=2)$. Because environmental variation was experienced among generations, we used the geometric mean fitness approach (Lewontin and Cohen, 1969; Leon, 1993). The geometric mean fitness of a genotype under fluctuating selection is:

$$
M\left(\mu_{i}\right)=w\left(\mu_{i, 1}\right)^{p} \cdot w\left(\mu_{i, 2}\right)^{1-p}
$$

For each value of $p$ and $A$, we integrated out Equation (1) over a range of possible phenotype distributions (assuming $\sigma=1$ for phenotypic error, using $\gamma$ and its modified forms as the fitness functions). Once the within-generation fitnesses were found for a given phenotype distribution (i.e. for a given genotype), we calculated the geometric mean fitness for each genotype from Equation (3). We then found $\mu^{*}$ by a root procedure to find the value of $\mu$ that maximized Equation (3) (i.e. where the first derivative of $M\left(\mu_{i}\right)$ was zero and the second derivative was negative). To determine the sensitivity of the models to phenotypic error, we created standard models $(p=0.5, A=0.5$ or 0.1 ) and tested each with $\sigma$ ranging from 0.5 to 3 . For visual reference, $\sigma=1$ in Fig. $2 \mathrm{~A}$. 


\section{Results}

\section{Within-generation optima}

The phenotypic optimum in all environments was at $z^{*}=1.0$. The genetic optimum in the default environment was $\mu^{*}=1.48$ (with $\sigma, \sigma^{2}=1$ ). Thus, the most fit genotype in this environment specified a mean phenotype that was $48 \%$ greater than the optimal within-generation phenotype. This was expected based on previous models (Yoshimura and Shields, 1987, 1992, 1995) because the fitness function drops off rapidly for values of $z<1$, so the optimal phenotype distribution was located away from the sharp drop to hedge against the probability of error to the left, where fitness was lower than the equivalent error to the right.

\section{PFC model}

All PFC modified environments $(0<A \leq 1,0 \leq p \leq 1, \sigma=1)$ had the same genetic optimum as the default environment $\left(\mu^{*}=1.48\right)$. Thus, the genetic optimum with environmental fluctuation between PFC environments did not change, regardless of the frequency of each environment. The evolutionarily optimal genotype, therefore, does not depend on environmental fluctuation of the proportionate-fitness-change type. The rate of evolution (i.e. the strength of directional and stabilizing selection) towards $\mu^{*}=1.48$ will be constant for any PFC selection regime because the relative fitness structure among phenotypes stays the same. In the example given previously, evolution of body size in an annual plant would occur at the same rate, and towards the same trait value, in fire-prone and fire-safe years.

\section{AFC (fitness threshold) model}

The genetic optimum (i.e. the optimal phenotype distribution) in the AFC models depended on the degree to which the fitness threshold was raised and the proportion of generations experiencing modified environments. For the examples depicted in Fig. $2 \mathrm{C}$, the within-generation genetic optima (from Equation 1) were $\mu^{*}=1.48$ in the good environment, $\mu^{*}=1.34$ in the moderate environment and $\mu^{*}=1.11$ in the harsh environment. Thus, raising the fitness threshold moved the genetic optimum towards the phenotypic optimum $\left(\mu^{*} \rightarrow z^{*}\right)$, whereas $\mu^{*}$ diverged from $z^{*}$ when the fitness threshold was lowered.

Genetic optima for environmental fluctuation between the good environment and the full range of AFC modified environments are illustrated in Fig. 4. The genetic optimum for each AFC model differed even though the source of fitness reduction was unrelated to the trait $z$ and despite the fact that the phenotypic optimum never deviated from $z^{*}=1$. All that changed about the environments in these models was that the fitness threshold was raised due to phenotype- and genotypeindependent changes in absolute fitness. Raising the fitness threshold makes the relative fitness landscape for phenotypes more steeply sloped, and should speed evolution by increasing the strength of directional and stabilizing selection.

Uncommon harsh environments $(p>0.8)$ had little effect on the genetic optimum, unless the area below the harsh environment curve was small $(A<0.2$; Fig. 4). Thus, only common or catastrophic environmental stresses would keep the population mean phenotype near the phenotypic optimum (in our example, $z^{*}=1$ ). Otherwise, the population mean would be closer to the genetic optimum in a good environment (i.e. $\mu_{j=1}{ }^{*}=1.48$ ).

In the example of the fish given previously, evolution of jaw morphology would proceed differently in harsh- and mild-winter years: the relative fitness structure among phenotypes would change and alternative genetic optima would hold each winter. 


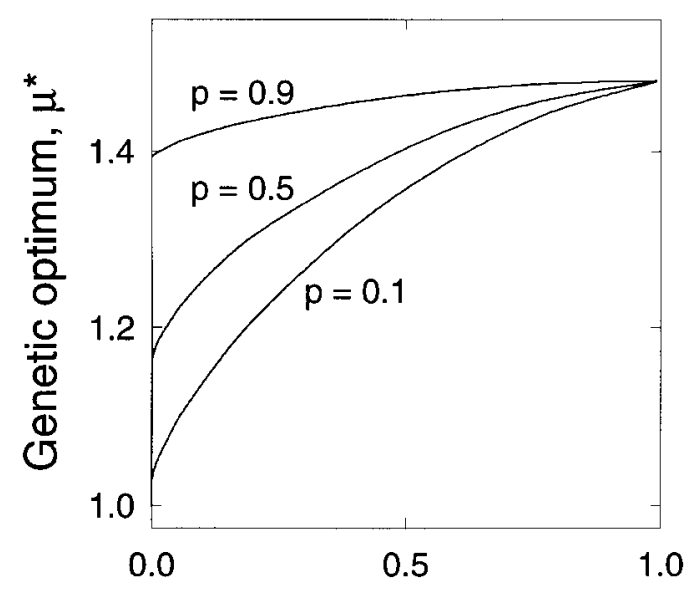

Fitness proportion, $A$

Figure 4. Effects of the frequency and intensity of absolute fitness changes on the genetic optima. $A$ is the area of the modified fitness function relative to the best environment, $\gamma$. The proportion of $\gamma$ environments is $p ; 1-p$ is the proportion of modified environments.

\section{Phenotypic error}

The sensitivity of shifts in genetic optima to phenotypic error are presented in Fig. 5. The peak shifts increased in magnitude logistically as phenotypic error increased. The shifts became nontrivial at low phenotypic errors $(\sigma=0.5)$.

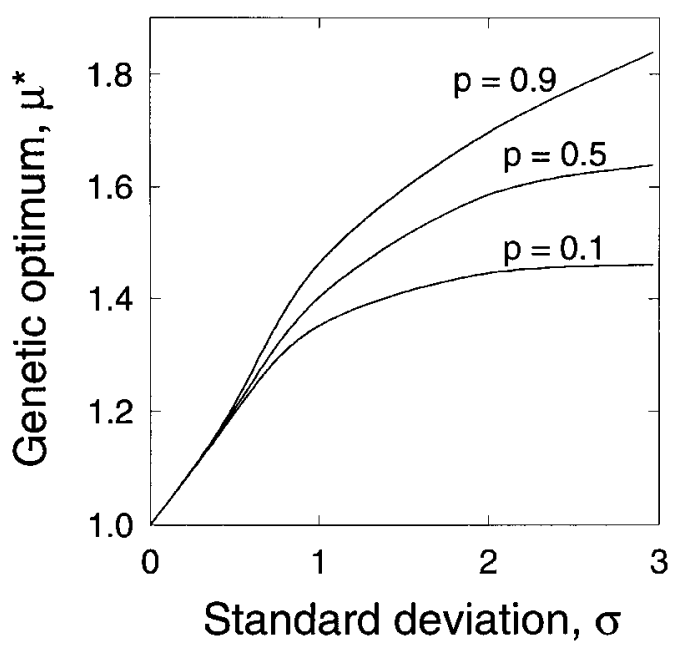

Figure 5. Sensitivity analysis for phenotypic error, assuming $A=0.5$. The magnitude of the peak shift from the phenotypic optimum increased logistically as phenotypic error increased. The top line represents infrequently harsh environments, the bottom line represents mostly harsh environments, and the middle line represents equal occurrences of harsh and good environments. 


\section{Discussion}

\section{Evolutionary implications}

The forms of environmental variation that we defined seem to us like biologically common occurrences. Studies that report fitness functions over multiple generations are necessary to elucidate which forms of environmental variation are more common. In perhaps the largest published study, Weis et al. (1992) reported fitness functions over four consecutive years for gall-size in a univoltine fly (Eurosta solidago). The data composing the functions suggest a combination of PFC and AFC among the four generations studied. For example, the small fitness peak for galls between 7 and 13 $\mathrm{mm}$ appears to submerge below a fitness threshold in the harshest year (1987-88), suggesting large absolute fitness declines in that year $(A \approx 0.5)$. As predicted by single-generation models of probabilistic optimization (Yoshimura and Shields, 1987, 1992, 1995), the most common gall-size phenotype was consistently located in the skewed end of the fitness function tail, not at the peak. The success of single-generation analyses using probabilistic optimization is encouraging, yet single-generation estimates may not be adequate to predict evolution in fluctuating environments (Levins, 1968).

Ecologists often assume that multiple measures of fitness functions can be averaged to describe selection forces if a basic fitness function exists for phenotypes that only fluctuate due to environmental noise. Evolutionary theorists assume that only genotype-dependent variation in fitness alters evolution in a systematic way. One model of random environmental change (PFC) supported these views, whereas the other (AFC) was exceptional. Proportionate fitness change did not alter the adaptive landscape because differences in relative fitness remained intact as the environment shifted. Absolute fitness change affected the adaptive landscape in two ways: (1) the peaks (genetic optima) changed location and (2) the slopes defining the peaks changed. Lowered fitness thresholds resulted in low-intensity selection away from phenotypic optima. Raised thresholds resulted in intense selection for genetic optima closer to phenotypic optima. Thus, infrequently raised fitness thresholds (catastrophic years) will affect evolutionary outcomes more than infrequently lowered thresholds (boom years).

The evolutionary scenario presented in this paper is one of shifting genetic optima among generations with no necessary shift in phenotypic optima. Although we can calculate the crossgenerational genetic optimum for fluctuating environments, $\mu^{*}$, it is important to remember that selection acts against this genotype, in favour of a different alternative each generation. That is, $\mu^{*}$ is most fit on average, but it is rarely the optimum within any one generation. Genetics is always playing evolutionary catch-up, because the progress made up a peak in one generation of selection may be a liability when the environment shifts. The shifts in genetic optima will have the effect of maintaining genetic variance, just as with models in which phenotypic optima fluctuate (Dempster, 1955; Haldane and Jayakar, 1963). In the fitness threshold model, however, genetic optima vary while the phenotypic optimum remains constant. Such constancy, therefore, cannot be used to predict evolutionary stasis.

Another implication of genetic optima shifting among generations is that a reaction norm could evolve. In this case, the optimal plastic genotype would produce alternative phenotype distributions based on cues indicating the position of the fitness threshold. This would be an unusual form of phenotypic plasticity because the environmental state used to cue alternative developmental pathways would have no relationship to the optimal phenotype. Adaptive phenotypic plasticity is generally thought of in terms of coarse-grained environmental fluctuation, in which the phenotype changes among generations based on cues that indicate the optimal phenotype (Levins, 1968; Via and Lande, 1985; Leon, 1993). 
The fitness threshold model rests on the idea that fitness functions and phenotype distributions are shaped differently. We did not allow phenotype distributions to evolve in our models, although it is clear that the stabilizing selection we assumed would favour a genotype producing $z^{*}$ with no developmental error $\left(\sigma^{*}=0\right)$. Perfect canalization, however, is likely to be uncommon for quantitative traits in natural circumstances. For example, developmental error may be necessary to increase genotypic environmental tolerance (Bull, 1987; Gabriel and Lynch, 1992), or could be unavoidable (Waddington 1957), or could be costly (sensu phenotypic plasticity; DeWitt et al., 1998). Also, if a locus determining the focal trait had epistatic interactions with other loci, then species with genetic recombination would always have phenotypic variation around the average effect of a given allele (Falconer and Mackay, 1996). If phenotypic error is necessary, inevitable or costly, then asymmetric canalization of that error may be the next best adaptation to altering the amount of error. For example, it may be beneficial to skew developmental error towards the skewed end of the fitness function. This proposition may explain why the phenotype distributions observed by Weis et al. (1992) each year were skewed like the fitness functions. The nature and importance of developmental skewness is a largely unexplored area of evolutionary biology. Although methods exist to estimate selection on phenotypic variance at the population level (Lande and Arnold, 1983), the extension to higher moments of individual phenotype distributions has yet to be developed.

\section{Empirical implications}

An important implication of the fitness threshold model is that we can expect apparently maladaptive evolution. For example, Parker (1991) demonstrated apparent maladaptive evolution of disease resistance in the annual legume Amphicarpaea bracteata. Disease resistance declined in a population despite the constant presence of the pathogen. He attributed this to genetic hitchhiking (i.e. correlated selection) after he was able to rule out known alternative hypotheses, such as gene flow and genetic drift. The present model provides another possible explanation for apparently maladaptive evolution. Evolution towards a phenotypic optimum value should occur in harsh years (i.e. years with high fitness thresholds) but evolution away from peaks should occur in good years (i.e. low fitness thresholds). Thus, genetic correlations (adaptive trade-offs) cannot be the de facto explanation when evolution away from a phenotypic fitness peak is documented.

What this also means is that measuring a fitness function in a single generation is not sufficient to calculate optima. The magnitude of displacement between genetic and phenotypic optima depends on the amount and type of inter-generational fluctuation. For example, Roff (1981) computed a fitness function for body size in Drosophila melanogaster using empirical data. He found that the single-generation phenotypic optimum differed by approximately $7.5 \%$ from the mean phenotype produced. Recently, Yoshimura and Shields (1995) explained a fraction of this discrepancy by using probabilistic optimization, taking into account a rough estimate of phenotypic error by Mangan (1978). The discrepancy could be further resolved if the fitness threshold in Roff's calculations was for any reason lower than the natural value, which could easily occur under laboratory conditions with ad libitum food.

\section{Practical implications}

The genetic peak shifts caused by changes in the fitness threshold may also bear on the management of natural populations. Management techniques often lower fitness thresholds independent of organismal phenotypes and genotypes. For example, pesticide use, supplementary feeding and other practices may lower fitness thresholds. These practices may also inflate the fitness functions as in our PFC model - but our model shows that this would have no bearing on what trait values 
evolve by natural selection. In contrast, if the fitness threshold is lowered by management practices, genetic optima will be driven away from the natural peak for the population. Thus, when organisms are reintroduced to natural conditions - the goal of many management programmes - they will express maladapted (overly risk-averse) phenotypes. Thus, if the natural environment crashes after reintroduction, the risk-prone phenotypes needed for persistence of the population may not exist and the previously managed population could go extinct.

The implications of this model are far-reaching and cannot be fully explored here. Many formerly unexplained examples of seemingly suboptimal traits or maladaptive evolution can be addressed by stochastic models that incorporate phenotypic error within generations (e.g. pollen dispersal in plants, clutch size in birds, body size in Drosophila, gall size in Eurosta). We may likewise be able to expand our explanatory power by including even random inter-generational environmental variance in our models and empirical work. The present models suggest that environmental fluctuation involving shifted fitness thresholds changes both the location of genetic optima and the rate of evolution towards optima. Thus, what have often seemed like random population booms and busts may be interpretable as fluctuating fitness thresholds, and hence may be evolutionarily important as suggested by early ecological geneticists such as Ford (1964). Ford and others argued that supplemental feeding and easing of general environmental conditions allows a greater range of phenotypes to survive and increases genetic variation. Based on our models, we concur so long as the form of environmental favourability involves lowered fitness thresholds, rather than proportionate fitness increases.

\section{Acknowledgements}

We thank the EEB groups at Binghamton University and the University of Kentucky, as well as our anonymous reviewers, for valuable commentary. We thank in particular D.S. Wilson, B.W. Robinson and J.D. Arendt for extended discussions on this topic.

\section{References}

Boyce, M.S. and Perrins, C.M. (1987) Optimizing great tit clutch size in a fluctuating environment. Ecology 68 , $142-153$.

Brodie, E.D. III, Moore, A.J. and Janzen, F.J. (1995) Visualizing and quantifying natural selection. Trends Ecol. Evol. 10, 313-318.

Bull, J.J. (1987) Evolution of phenotypic variance. Evolution 41, 303-315.

Bulmer, M.G. (1984) Delayed germination of seeds: Cohen's model revisited. Theor. Pop. Biol. 28, 263-287.

Dempster, E.R. (1955) Maintenance of genetic heterogeneity, Cold Spring Harbor Symposium. Quant. Biol. 20, 25-32.

DeWitt, T.J., Sih, A. and Wilson, D.S. (1998) Costs and limits of phenotypic plasticity. Trends Ecol. Evol. 13, $77-81$.

Dobzhansky, T. (1970) Genetics of the Evolutionary Process. Columbia University Press, New York.

Falconer, D.S. and Mackay, T.F.C. (1996) Introduction to Quantitative Genetics, 4th edn. Longman, Essex.

Fisher, R.A. (1930) The Genetical Theory of Natural Selection. Oxford University Press, Oxford.

Ford, E.B. (1964) Ecological Genetics. Chapman \& Hall, London.

Gabriel, W. and Lynch, M. (1992) The selective advantage of reaction norms for environmental tolerance. J. Evol. Biol. 5, 41-59.

Haldane, J.B.S. and Jayakar, S.D. (1963) Polymorphism due to selection of varying direction. J. Genetics 58, 237-242.

Hassell, M.P. (1978) The Dynamics of Arthropod Predator-Prey Systems. Monographs in Population Biology No. 13. Princeton University Press, Princeton, NJ. 
Lande, R. and Arnold, S.J. (1983) The measurement of selection on correlated characters. Evolution 37, 12101226.

Leon, J.A. (1993) Plasticity in fluctuating environments. Lecture Notes in Biomathematics 98, 105-121.

Levins, R. (1968) Evolution in Changing Environments. Princeton University Press, Princeton, NJ.

Lewontin, R.C. and Cohen, D. (1969) On population growth in a randomly varying environment. Proc. Nat. Acad. Sci. USA 62, 1056-1060.

Mangan, R.L. (1978) Comparative interactions among host plant specific Drosophila species. PhD dissertation, University of Arizona, Tucson, AZ.

Mayr, E. (1970) Populations, Species, and Evolution. Harvard University Press, Cambridge, MA.

Mountford, M.D. (1968) The significance of litter-size. J. Anim. Ecol. 37, 363-367.

Oksanen, L. and Lundberg, P. (1995) Optimization of reproductive effort and foraging time in mammals: The influence of resource level and predation risk. Evol. Ecol. 9, 45-56.

Parker, M.A. (1991) Nonadaptive evolution of disease resistance in an annual legume. Evolution 45, 12091217.

Roff, D. (1981) On being the right size. Am. Nat. 118, 405-422.

Schmalhausen, I.I. (1949) Factors of Evolution: The Theory of Stabilizing Selection. Blakiston, Philadelphia, PA.

Seger, J. and Brockmann, H.J. (1987) What is bet-hedging? Oxford Surv. Evol. Biol. 4, 182-211.

Slatkin, M. and Lande, R. (1976) Niche width in a fluctuating environment - Density independent model. Am. Nat. 110, 31-55.

Via, S. and Lande, R. (1985) Genotype-environment interaction and the evolution of phenotypic plasticity. Evolution 39, 505-522.

Waddington, C.H. (1957) The Strategy of the Genes. Macmillan, New York.

Weis, A.E. and Gorman, W.L. (1990) Measuring canalizing selection on reaction norms: An exploration of the Eurosta-Solidago system. Evolution 44, 820-831.

Weis, A.E., Abrahamson, W.G. and Andersen, M.C. (1992) Variable selection on Eurosta's gall size. I: The extent and nature of variation in phenotypic selection. Evolution 46, 1674-1697.

Yoshimura, J. and Shields, W.M. (1987) Probabilistic optimization of phenotype distributions: A general solution for the effects of uncertainty on natural selection? Evol. Ecol. 1, 125-138.

Yoshimura, J. and Shields, W.M. (1992) Components of uncertainty in clutch-size optimization. Bull. Math. Biol. 54, 445-464.

Yoshimura, J. and Shields, W.M. (1995) Probabilistic optimization of body size: A discrepancy between genetic and phenotypic optima. Evolution 49, 375-378. 